\title{
Métodos de pesquisa mistos e revisões de literatura mistas: conceitos, construção e critérios de avaliação
}

\author{
Mixed methods research and mixed reviews of literature: concepts, design, and evaluation \\ criteria
}

\author{
Maria Cristiane Barbosa Galvão \\ Doutora em Ciências da Informação pela Universidade de Brasília - UnB. \\ Professora da Faculdade de Medicina de Ribeirão Preto da Universidade de São Paulo - USP. \\ E-mail: mgalvao@usp.br \\ Pierre Pluye \\ Professor Titular da Faculty of Medicine da McGill Unversity, Canadá \\ E-mail: pierre.pluye@ mcgill.ca
}

Ivan Luiz Marques Ricarte Doutor em Engenharia Elétrica pela University System of Maryland, Estados Unidos. Professor Titular da Faculdade de Tecnologia da Universidade Estadual de Campinas - UNICAMP.

E-mail: ricarte@unicamp.br

\section{Resumo}

O emprego de métodos mistos e revisões de literatura mistas pode ser uma janela de oportunidades para que os pesquisadores se insiram no processo de internacionalização da ciência. Desse modo, o presente trabalho sistematiza conceitos, formas de construção e critérios de avaliação relacionados a pesquisas empregando métodos mistos e revisões de literatura mistas. No que se refere à metodologia, empregou-se uma revisão de literatura seletiva, considerando o critério de relevância do texto para que se tenha uma inicialização e compreensão dos aspectos essenciais relacionados a métodos mistos e revisões mistas. Em seguida, empregou-se um relato de caso que apresenta a percepção de pesquisadores brasileiros sobre uma experiência de ensino-aprendizagem com a temática de métodos mistos e revisões mistas. Como resultado, entende-se que o conhecimento sobre métodos de pesquisa mistos e revisões de literatura mistas tem potencial para aumentar a capacidade de investigação nacional e internacional; ampliar a massa crítica de revisores para a atuação em agências de fomento e em periódicos nacionais e internacionais; e, reforçar a colaboração internacional para desenvolvimento de projetos de pesquisa.

Palavras-chave: Métodos de pesquisa mistos. Revisões de literatura mistas. Educação continuada em pesquisa. Ciência da informação. Avaliação de pesquisa.

\begin{abstract}
Mixed methods and mixed literature reviews can provide a window of opportunity for researchers to enter in the process of science internationalization. In this paper, we systematize concepts, design, and evaluation criteria related to research using mixed methods and mixed literature reviews. Initially, we performed a selective literature review, considering the criteria of text relevance in order to have an initial understanding of essential aspects of mixed methods and mixed reviews. Then, we present a case report showing the perception of Brazilian researchers on a teaching and learning experience on mixed methods and mixed reviews. As a result, we understand that knowledge on mixed research methods and mixed literature reviews has the potential to increase the capacity of national and international research; increase the critical mass of reviewers for the work in development agencies and national and international journals; and strengthen international cooperation for the development of research projects.
\end{abstract}

Keywords: Mixed methods research. Mixed literature reviews. Continuing education research. Information Science. Research evaluation. 


\section{Introdução}

Vivemos em uma época de problemas complexos que envolvem sistemas repletos de incertezas sociais e institucionais, para os quais não há clareza sobre a sua natureza e para os quais está se esgotando o tempo para encontrar soluções. Tais problemas se referem a temáticas como desigualdades de poder, violações dos direitos humanos, degradação ambiental, alterações climáticas, pobreza, falta de acesso a serviços de saúde, educação e informação, desigualdade social e econômica, instabilidade geopolítica, migração, repatriamento forçado, violência, discriminação e opressão de todos os tipos e dimensões, pessoas com deficiência, minorias étnicas e raciais. Para lidar com essa complexidade, os desafios para os pesquisadores vão desde a formação de equipes multidisciplinares para partilhar conhecimentos de forma respeitosa até a busca por inovações nas abordagens metodológicas que possam melhor encaminhar essas questões. É a partir desse cenário que se entende que os métodos mistos e a criatividade inerente em como usá-los podem aumentar o potencial de mudança transformadora e não agregadora de danos adicionais ao contexto delineado (MERTENS et al., 2016).

As questões complexas não são exclusividade da sociedade, também se manifestam no campo científico nela integrado. Em que pese algumas políticas locais acadêmicas propagarem que a internacionalização da ciência deriva da vontade ou da dedicação individual do pesquisador, estudos apontam que há nesse processo elementos como: a existência de barreiras geopolíticas e culturais que impedem a internacionalização (O'CONNOR, 2012); o isolamento científico de alguns países em decorrência de políticas e interesses locais de pesquisa (LADLE; TODD; MALHADO, 2012); e parâmetros de revisão de manuscritos adotados por periódicos internacionais que priorizam critérios metodológicos que não são necessariamente enfatizados em alguns contextos locais (SPIGT; ARTS, 2010; BORNMANN et al 2012; WINCK et al, 2011).

Pelo exposto, este texto parte do pressuposto de que o emprego dos métodos mistos em pesquisas científicas traz, ao menos, duas janelas de oportunidade para pesquisadores.

A primeira janela de oportunidades se filia ao fato dos métodos mistos viabilizarem o estudo de problemas complexos e a construção de resultados de pesquisa potencialmente mais completos e relevantes.

E aqui se pode citar como exemplo o estudo de Yi (2015), Health literacy and health information behavior of Florida public library users: A mixed methods study, que coloca 
questões como: Qual é o estado atual do letramento em saúde dos usuários que frequentam bibliotecas públicas? Há correlação entre o letramento em saúde dos usuários e suas características demográficas? Como os usuários da biblioteca pública percebem sua capacidade de buscar informações sobre saúde em bibliotecas públicas? Como encontram recursos de informação de saúde? Como percebem sua capacidade de avaliar informações de saúde? Como avaliam as informações de saúde? Como percebem sua capacidade de usar informações de saúde? Como eles usam informações de saúde? Quais são as barreiras para encontrar, avaliar e utilizar informações de saúde encontradas pelos usuários de bibliotecas públicas? É possível perceber que esse estudo traz questões de pesquisa tanto de natureza qualitativa como de natureza quantitativa e, consequentemente, para responde-las, Yi empregou métodos mistos. $\mathrm{Na}$ primeira fase, quantitativa, 200 questionários foram aplicados a usuários de bibliotecas públicas. Na sequência, na fase qualitativa do estudo, 20 dos usuários que responderam ao questionário foram entrevistados empregando-se técnica de incidente crítico. Entre os muitos achados do estudo, o autor aponta que uma das barreiras para o uso da informação em saúde em bibliotecas públicas é a localização da informação. Dessa forma, recomenda que, para contribuírem de forma mais efetiva com a saúde pública, as bibliotecas públicas devem colocar informações em saúde em lugares mais estratégicos como, por exemplo, próximo à porta de entrada da biblioteca, na recepção da biblioteca e em lugares onde os usuários da biblioteca costumam descansar (YI, 2015).

A segunda janela se filia à oportunidade dos pesquisadores se inserirem no processo de internacionalização da ciência, pois a aplicação dos métodos mistos é ainda insipiente no contexto global, embora apresente uma tendência de crescimento, conforme pode ser observado em diferentes bases de dados.

Considerando a base de dados multidisciplinar Scopus, de abrangência internacional, em busca realizada em 13 de julho de 2016 empregando-se a expressão exata "mixed method" no campo título, encontrou-se um total de 4.064 artigos, dos quais 486 produzidos em 2016, 793 em 2015, 661 em 2014, 474 em 2013, 401 em 2012, 314 em 2011, 227 em 2010, 187 em 2009, 137 em 2008, 54 em 2007, 62 em 2006, e assim sucessivamente, até chegar-se ao ano de 1977 com 6 artigos envolvendo métodos mistos.

Já a busca por estudos empregando métodos mistos em uma base de dados em Ciência da Informação, Library and Information Science Abstracts (LISA), realizada em 13 de julho de 2016 empregando-se a expressão exata "mixed method" no campo título, encontraram-se 7 
artigos, produzidos entre 2005 e 2014, que empregam métodos mistos. Eles foram publicados pelos periódicos: International Journal of Information Management, New Review of Academic Librarianship, Serials Librarian, The American Archivist, Evidence Based Library and Information Practice, e Information, Communication \& Society.

$\mathrm{Na}$ base de dados Scientific Electronic Library Online (SCIELO), que congrega expressiva produção da América Latina, em busca realizada em 13 de julho de 2016, foi encontrado apenas 1 artigo empregando métodos mistos publicado em 2015, na Revista Brasileira de Ciências Sociais.

Esse exercício ilustra, de um lado, a tendência de crescimento na publicação de artigos envolvendo métodos mistos no cenário internacional, bem como o caminho que pesquisadores brasileiros e latino-americanos precisam percorrer. Especificamente no campo da ciência da informação, considerando que o objeto informação possui dimensões objetivas e subjetivas, o conhecimento dos métodos mistos pode beneficiar as pesquisas desenvolvidas na área, permitindo melhor compreensão dos fenômenos relacionados a esse objeto (FIDEL, 2008; MA, 2012).

Considerando as janelas de oportunidade apresentadas, o presente artigo sistematiza conceitos, formas de construção e critérios de avaliação relacionados a pesquisas empregando métodos mistos e revisões de literatura mistas, sendo composto pelas seguintes partes: métodos de pesquisa mistos (conceitos, construção e avaliação); revisões de literatura mistas (conceitos, construção e avaliação); ensino de métodos mistos e revisões mistas para pesquisadores; e conclusão. No que se refere à metodologia, este trabalho empregou uma revisão de literatura seletiva. Para a seleção dessa literatura empregou-se o critério de relevância do texto para que se tenha uma compreensão dos aspectos essenciais relacionados a métodos mistos e revisões de literatura mistas. Em seguida, empregou-se o relato de caso que apresenta a percepção de pesquisadores brasileiros quanto a uma experiência de ensino-aprendizagem com a temática de métodos mistos e revisões mistas. Dessa maneira, entende-se que o presente texto segue uma abordagem pragmática, qualitativa e exploratória. 


\section{Métodos de pesquisa mistos: conceitos, construção e avaliação}

Pesquisas com métodos qualitativos fornecem descrições detalhadas de fenômenos complexos, incluindo seus aspectos contextuais, ou focam em análises aprofundadas envolvendo poucos indivíduos. Desse modo, seus resultados não são generalizáveis. Já, as pesquisas com métodos quantitativos costumam examinar a associação entre variáveis que podem ser generalizadas para uma população por meio de inferências estatísticas. Focam na análise de grandes amostras, porém seus achados não levam à compreensão de processos individuais.

A pesquisa com métodos mistos combina os métodos de pesquisa qualitativos e quantitativos e tem por objetivo generalizar os resultados qualitativos, ou aprofundar a compreensão dos resultados quantitativos, ou corroborar os resultados (qualitativos ou quantitativos). Uma pesquisa que empregue métodos mistos, geralmente, é desenvolvida por um grupo de pesquisadores que possuem diferentes habilidades e competências em pesquisa e podem aplicar com coerência e precisão diferentes métodos (JOHNSON; ONWUEGBUZIE; TURNER, 2007; PLUYE, 2012).

Por convenção, a pesquisa envolvendo métodos mistos deve, na medida do possível, explicitar tal característica no título do artigo. Assim, tem-se, a seguir três exemplos de trabalhos que apresentam a expressão "métodos mistos" em seus títulos:

Prevalence, motivations, and social, mental health and health consequences of cyberbullying among school-aged children and youth: protocol of a longitudinal and multi-perspective mixed method study (MISHNA et al., 2016).

Using a sequential exploratory mixed-method design to examine racial hyperprivilege in higher education (CABRERA, 2011).

Information literacy instruction assessment and improvement through evidence based practice: a mixed method study (WAKIMOTO, 2010).

O uso de métodos mistos, comumente, transcende o conhecimento disciplinar e se pauta no trabalho coletivo de dois ou mais pesquisadores. Assim, em linhas gerais, se a competência do pesquisador $A$ reside em pesquisa de caráter qualitativo, para desenvolver um estudo misto, ele deve considerar a possibilidade de se associar a um pesquisador $B$ que, por sua vez, tenha competência em pesquisa de caráter quantitativo. Dito de outra forma, é difícil que um 
pesquisador isoladamente possua todas as competências necessárias para o desenvolvimento de pesquisas desse caráter.

Embora ao longo da história da ciência alguns pesquisadores tenham tentado intuitivamente e individualmente combinar métodos qualitativos e quantitativos, a forma de integração/combinação de métodos de pesquisa tem sido cada vez mais criticamente estudada, sistematizada, passando gradativamente a ser acompanhada por um aporte conceitual crescente relacionado ao assunto.

Os principais métodos qualitativos que são integrados na construção de uma abordagem mista de pesquisa são: etnografia; fenomenologia; narrativas (biografias, relatos de vida); teoria fundamentada; estudo de caso e descrição qualitativa. Já os métodos quantitativos mais usados em abordagens mistas incluem: estudo controlado randomizado; ensaio clínico controlado; estudo de coorte; comparação de estudo de caso; séries temporais e pesquisa transversal analítica; estudo de prevalência; estudo de incidência; pesquisa transversal descritiva e série de casos.

No campo das ciências sociais, Bryman (2006), ao realizar uma revisão de literatura que abrangeu 232 artigos relacionados ao uso de métodos mistos, concluiu que o tipo de pesquisa empregando entrevista estruturada tende a predominar entre as técnicas quantitativas e que o tipo de pesquisa empregando entrevista aberta ou semi-estruturada tende a predominar no que diz respeito às técnicas qualitativas. Segundo esse estudo, as técnicas de coleta de dados predominantes, no campo das ciências sociais, em relação aos métodos mistos são: as entrevistas individuais não estruturadas, semi-estruturadas e estruturadas, grupos focais, questionários com ou sem opções de resposta, observação participante etnográfica e revisão da literatura. Em relação às técnicas de análise de dados associados com métodos mistos estão a análise de conteúdo, a análise temática e as análises estatísticas. É importante destacar que ao se referir à análise de conteúdo, Bryman (2006) aborda a quantificação de dados qualitativos, tais como a quantificação das respostas dadas por entrevistados.

O componente qualitativo de um método misto pode ser usado, por exemplo, para se conhecer ou compreender os aspectos culturais, econômicos, organizacionais, políticos e sociais de um fenômeno ou problema, bem como para descobrir possíveis variáveis que interferem em alguns contextos e não em outros. Por sua vez, o componente de caráter quantitativo pode medir as associações entre diferentes fatores e a magnitude de seus efeitos ou implicações. Para os leitores menos familiarizados com a terminologia de métodos de pesquisa, sugere-se a consulta de Schwandt (2007), que apresenta uma terminologia detalhada 
Maria Cristiane Barbosa Galvão, Pierre Pluye e Ivan Luiz Marques Ricarte Métodos de pesquisa mistos e revisões de literatura mistas: conceitos, construção e critérios de avaliação

relacionada aos métodos qualitativos, e de Porta (2008), que apresenta uma terminologia detalhada relacionada aos métodos quantitativos.

No que se refere à denominação de tipos de pesquisa empregando métodos mistos, Creswell e Clark (2010) propõem três termos adotados internacionalmente, quais sejam:

- Estudo exploratório sequencial, representado pela expressão QUAL>QUAN. É aquele que se inicia por uma etapa qualitativa e que, por sua vez, é seguida por uma etapa quantitativa. Nesse caso, os resultados quantitativos são mobilizados para confirmar ou generalizar os resultados qualitativos. Exemplo:

Alternative perspectives of safety in home delivered health care: a sequential exploratory mixed method study (JONES, 2016).

- Estudo explanatório sequencial, representado pela expressão QUAN>QUAL. É aquele que se inicia por uma etapa quantitativa e que, por sua vez, é seguida por uma etapa qualitativa. Nesse caso, os resultados qualitativos são mobilizados para interpretar ou explicar os resultados quantitativos. Exemplo:

Iranian adolescents' insufficient physical activity: a mixed methods explanatory sequential study (BAHEIRAEI et al., 2015).

- Estudo de convergência, representado pela expressão QUAN+QUAL. É aquele em que as etapas qualitativas e quantitativas são concomitantes, ou seja, os métodos qualitativos e quantitativos documentam os mesmos fenômenos de forma complementar, havendo, no momento da análise dos dados, uma integração dos resultados a fim de melhor entender o fenômeno. Exemplo:

Determinants of primary school non-enrollment and absenteeism: results from a retrospective, convergent mixed methods, cohort study in rural western Kenya (KING et al., 2015).

Há diversas técnicas que podem ser utilizadas para a integração de dados ou resultados em um estudo misto. A técnica da transformação, por exemplo, consiste em traduzir dados qualitativos para valores que possam ser integrados com os dados quantitativos, ou em categorizar dados quantitativos de forma que eles possam ser integrados aos dados qualitativos. Outra possibilidade é a técnica da comparação, na qual os resultados qualitativos e quantitativos são analisados separadamente sendo integrados apenas no momento da interpretação. 
De forma geral, todas essas técnicas de integração poderão de algum modo destacar as discrepâncias entre os dados e os resultados das análises qualitativa e quantitativa, diante das quais os pesquisadores terão que adotar alguma decisão: assumir que os resultados são complementares (reconciliação); iniciar novos estudos para melhor entender os resultados discrepantes (iniciação); ou simplesmente observar que os resultados de um método são distorcidos ou não acrescentam nada ao conhecimento do fenômeno (exclusão) (PLUYE et al., 2009b).

Ressalta-se que qualquer integração de método é legítima, mas somente quando essa integração satisfaz a três condições: a integração compreende, pelo menos, um método qualitativo e um método quantitativo; cada método é usado rigorosamente e seguindo padrões cientificamente aceitos; e a integração compreende abordagens metodológicas, dados e resultados. No entanto, não se considera que um estudo emprega métodos mistos quando esses métodos e resultados são totalmente dissociados, sem qualquer abordagem explícita de integração.

No que diz respeito à avaliação da qualidade de estudos empregando métodos mistos para fins de publicação ou financiamento, existem alguns parâmetros a serem observados (O’CATHAIN; MURPHY; NICHOLL, 2008; PLUYE, 2011; PACE et al., 2012).

Pluye et al. (2011) apresentam a Mixed Methods Appraisal Tool (ferramenta para análise de métodos mistos) para avaliação crítica de estudos ou projetos empregando métodos mistos. Essa ferramenta contempla 40 itens que devem ser observados, dentre eles:

- O estudo possui uma questão qualitativa e uma questão quantitativa, ou uma questão mista explícita?

- Os dados coletados permitem endereçar a questão ou as questões de pesquisa?

- Os métodos mistos são relevantes para endereçar as questões e os objetivos qualitativos e quantitativos do estudo?

- A integração dos dados qualitativos e quantitativos é relevante para endereçar os objetivos ou questões do estudo?

- O estudo apresenta as limitações associadas com essa integração? 
- O estudo apresenta as divergências dos dados ou dos resultados qualitativos e quantitativos?

- Em relação aos aspectos quantitativos dos estudos mistos, estão explícitas a forma de recrutamento dos participantes de pesquisa; as variáveis e medidas realizadas em relação ao problema de pesquisa estudado; a forma de análise dos dados; e a adequação da análise em relação à amostra estudada?

- Em relação aos seus aspectos qualitativos, estão explícitas a relevância da amostra em relação à população estudada; a apresentação dos critérios de inclusão e exclusão dos sujeitos de pesquisa; a explicação dos motivos pelos quais indivíduos elegíveis não foram incluídos no estudo?

- Em relação aos aspectos mistos propriamente ditos, estão explícitos a explanação dos motivos que levaram à adoção dos métodos mistos para estudar o problema de pesquisa proposto; a integração dos dados quantitativos e qualitativos e seu endereçamento substancial ao problema de pesquisa; como os dados foram integrados e quem participou dessa integração; as limitações advindas pelo emprego dos métodos mistos?

$\mathrm{Na}$ experiência dos autores do presente estudo, equipes multidisciplinares formadas por cientistas da informação ou de outras ciências humanas, da informática e da estatística são fundamentais para o êxito da pesquisa empregando métodos mistos, pois grandes volumes de dados e informações de diferente natureza precisam ser localizados, organizados e sistematizados, requerendo competências específicas desses cientistas e profissionais.

\section{Revisões de literatura mistas: conceitos, construção e avaliação}

Em todas as pesquisas, a revisão da literatura é um elemento essencial que permite especificar os objetivos em termos do que já é conhecido, ou discutir os resultados e significados das pesquisas anteriores e seus impactos no campo científico, na sociedade ou em um contexto mais específico.

São bem conhecidas as metassínteses de estudos qualitativos, as metaetnografias e as revisões sistemáticas de estudos experimentais controlados randomizados (com ou sem metanálise). No entanto, conhecem-se menos as chamadas revisões mistas, ou seja, que incluem a análise de estudos que empregaram métodos quantitativos, qualitativos ou métodos mistos 
(PLUYE et al, 2009a).

Embora com produção rara ao longo do século XX, as revisões mistas têm se tornado populares mais recentemente e algumas delas têm sido publicadas em periódicos científicos relevantes, como o British Medical Journal, cujo fator de impacto em 2016 é de 19,967.

São elementos essenciais na revisão de literatura: formulação de uma questão que embase a revisão; modos de identificação de estudos relevantes e potenciais que possam integrar a revisão; modos de seleção de estudos relevantes para compor a revisão; a análise crítica da qualidade da metodologia de pesquisa dos estudos selecionados para compor a revisão; e a síntese dos resultados presente nos estudos selecionados para compor a revisão.

Quanto ao rigor metodológico, as revisões mistas podem ser classificadas em três tipos:

- Revisões mistas sistemáticas, aquelas que explicitam todos os elementos.

- Revisões mistas reproduzíveis, aquelas que explicitam elementos suficientes para viabilizar que outros pesquisadores refaçam o seu percurso de construção.

- Revisões mistas de conveniência, aquelas que não explicitam os elementos considerados em sua construção e não são reproduzíveis.

De forma geral, em teses de doutorado, em dissertações de mestrado, em projetos destinados a financiamento, não se faz necessário a elaboração de uma revisão sistemática, mas é fundamental a realização de uma revisão reproduzível. Quando se deseja elaborar uma revisão mista sistemática, pode-se empregar como parâmetro a Mixed Methods Appraisal Tool (PLUYE et al, 2011), que foi especificamente desenhada para facilitar a análise da qualidade metodológica de estudos que tenham seguido diferentes desenhos de pesquisa.

Como já assinalado, revisões de literatura, ao sintetizarem o conhecimento sobre um tópico e quando revelam falhas em estudos anteriores, dão suporte e justificam novas pesquisas de caráter primário. As revisões mistas, especificamente, permitem compreender fenômenos, problemas, programas ou intervenções complexos.

Em algumas situações, o pesquisador já pode iniciar sua revisão consciente de que será necessário considerar resultados qualitativos e quantitativos, definindo uma questão de revisão mista. Uma típica questão de revisão mista é: o que as evidências quantitativas e qualitativas dos estudos anteriores nos dizem sobre o tópico X? Em outras situações, o pesquisador precisa adequar sua questão de revisão inicial, não mista, em decorrência dos resultados obtidos em 
seus levantamentos bibliográficos.

A revisão mista é a revisão de literatura na qual o grupo de revisores identifica, seleciona, avalia e sintetiza estudos qualitativos, estudos quantitativos e estudos mistos. Estando em concordância com o desenho de métodos mistos, revisões mistas, quanto ao seu desenho e forma de síntese, podem ser classificadas como convergentes ou sequenciais, que por sua vez podem ser exploratórias ou explanatórias.

A revisão mista de convergência quantitativa é apropriada quando, nos estudos analisados, há um número grande de amostras ou participantes de pesquisa. Nesse caso, os resultados dos estudos qualitativos, quantitativos e de estudos empregando métodos mistos são transformados em achados quantitativos (variáveis ou valores). Nesse tipo de revisão, a maneira mais comum de transformação de dados é a análise de conteúdo, que é uma técnica para a simplificação de um fenômeno que reduz um grande número de dados em um número relativamente pequeno de categorias (POPE; MAYS; POPAY, 2007). Uma discussão mais detalhada sobre revisão mista de convergência quantitativa pode ser encontrada em Buelens et al. (2008).

Ressalta-se que o rigor de uma análise de conteúdo está baseado: na possibilidade de reprodução das categorias criadas, na confiabilidade da codificação (definição clara para cada categoria criada), e na concordância dos revisores na aplicação das categorias criadas. Essa concordância deve ser considerada estatisticamente, por exemplo, com base no índice Kappa (NEUENDORF, 2002).

A revisão mista de convergência qualitativa é adequada quando os estudos analisados possuem amostras pequenas e estão voltados para desenvolver, refinar e revisar um quadro conceitual, por exemplo. Nesse caso, os resultados dos estudos qualitativos, estudos quantitativos e de estudos empregando métodos mistos são transformados em achados qualitativos (por exemplo, em temas). A técnica mais comum empregada nessa transformação é a análise temática (podendo o tema ser uma categoria, um código ou um padrão) que descreve, organiza observações e interpreta aspectos de um fenômeno. (POPE; MAYS; POPAY, 2007). Um exemplo de revisão mista de convergência qualitativa pode ser encontrado em Bélanger et al. (2011).

O rigor de uma análise temática é baseado: na transposição do texto (dados coletados) para os temas e vice-versa; na discussão e consenso entre os revisores; e, quando necessário, na 
arbitragem de um terceiro revisor. Considerando a formulação da questão de revisão, há três abordagens para a realização da análise temática: a indutiva, com abordagem construída a partir da teoria, com temas derivados dos dados; a dedutiva, com abordagem dirigida por teoria, com temas pré-definidos e dados atribuídos a temas; e a híbrida, com temas pré-definidos, dados relacionados a temas, temas embasados por dados ou revisados por dados, ou com a presença de novos temas derivados de dados (BOYATZIS, 1998).

Um ponto importante no arcabouço terminológico internacional é que não se deve confundir análise de conteúdo (técnica quantitativa baseada em categorias que serão quantificadas) e análise temática (técnica qualitativa baseada na proposição de temas). Essa confusão pode ocorrer em alguns contextos.

A revisão mista sequencial exploratória é composta por duas etapas. Na etapa 1, os resultados dos estudos qualitativos, quantitativos e dos estudos empregando métodos mistos são transformados em achados qualitativos usando, por exemplo, a análise temática. Na etapa 2, quando há uma entidade comum entre os estudos quantitativos, os resultados quantitativos são tabulados e comparados. A interpretação dos achados na etapa 1 e na etapa 2 sugerem novas hipóteses e podem revelar falhas no conhecimento científico existente. Por exemplo, na etapa 1, pode-se explicitar uma tipologia. e, na etapa 2, podem-se explicitar indicadores ou medidas relacionados a cada tipo existente na tipologia. Na revisão mista sequencial exploratória, a integração de dados ocorre em dois momentos: entre a etapa qualitativa e a etapa quantitativa, ou seja, partindo-se dos achados da etapa 1 (síntese qualitativa) que fornece subsídios e elementos para a etapa 2 (síntese quantitativa). e na interpretação dos achados dessas duas etapas. Alguns exemplos simples dessa modalidade de revisão podem ser encontrados em Pluye et al. (2005) e Mills et al. (2006).

A revisão mista sequencial explanatória é empregada nos casos onde se quer medir os efeitos de ações, intervenções ou programas (etapa 1) e explicar diferenças em seus efeitos (etapa 2). Nessa modalidade de revisão, a integração ocorre entre as etapas quantitativa e qualitativa, na medida em que a síntese quantitativa (etapa 1) fornece subsídios para a síntese qualitativa (etapa 2), e na interpretação dos achados das duas etapas. Na etapa 1, os resultados dos estudos quantitativos e dos estudos empregando métodos mistos são tabulados, sendo medidas a presença e diferenças de determinados fenômenos. Na etapa 2, os resultados qualitativos e os dos estudos empregando métodos mistos são sintetizados em achados qualitativos. Então, a interpretação dos achados da etapa 1 e da etapa 2 sugerem a explicação e 
podem revelar falhas no conhecimento científico existente. Exemplos desse tipo de revisão podem ser encontrados nos estudos de Harden et al. (2009) e Thomas et al. (2004).

Ressalta-se que, para assegurar uma melhor qualidade da revisão de literatura mista, é necessário que um profissional da informação ou um pesquisador da ciência da informação integre a equipe de pesquisa e seja responsável pelo estabelecimento das metodologias de acesso à informação nas bases de dados internacionais, pela elaboração das estratégias de busca multilíngue, e que auxilie na validação dos critérios de seleção das informações que serão empregadas na pesquisa. Por tais razões, o conhecimento sobre as revisões de literatura mistas poderia integrar o ensino de graduação e pós-graduação nesse campo do conhecimento.

\section{O ensino de métodos mistos e revisões mistas para pesquisadores}

Conforme se pode observar nos parágrafos anteriores, o conhecimento de métodos mistos e revisões mistas não se dá de forma espontânea, sendo necessária uma educação continuada para que esse conhecimento seja transferido e assimilado pelos pesquisadores.

Considerando esse contexto, com base em quatro anos de experiência no ensino de métodos mistos e revisões mistas no Canadá e na Europa, realizou-se, no âmbito de uma universidade pública, um curso de métodos mistos e revisões mistas para pesquisadores brasileiros. O curso foi ministrado por professor especialista em métodos mistos.

A duração do curso compreendeu 5 dias (segunda-feira à sexta-feira), perfazendo um total de 40 horas, nos quais foram abordados os seguintes conteúdos:

- Introdução aos métodos mistos.

- Problemas, objetivos e questões apropriados aos métodos mistos.

- Desenho de pesquisa com métodos mistos.

- Combinação de dados quantitativos e qualitativos.

- Análise crítica de métodos mistos.

- Introdução à revisão de literatura mista.

- Problemas, objetivos e questões apropriadas às revisões mistas. 
- Revisão mista de convergência quantitativa.

- Revisão mista de convergência qualitativa.

- Revisão mista sequencial.

- 16 exercícios práticos.

- 8 horas de leitura individual e programada, contemplando aspectos teóricos e exemplos ilustrativos de estudos empregando métodos mistos e revisões mistas.

A divulgação do curso foi realizada, em língua portuguesa, na referida Universidade, bem como em jornais locais, redes sociais e listas de discussão de diferentes áreas do conhecimento, incluindo as do campo da ciência da informação, informática e saúde.

Foram adotados como prerrequisitos para participação no curso: ter realizado ao menos uma pesquisa de caráter qualitativo ou quantitativo (nível mestrado) e fluência na língua inglesa. Foram disponibilizadas 20 vagas gratuitas, considerando que um número excessivo de alunos não permitiria dar a atenção devida e específica ao interesse de cada participante.

Foram matriculados no curso 16 pesquisadores, sendo 4 doutores em enfermagem, 3 doutores em educação física, 2 mestres em enfermagem, 2 doutores em medicina, 1 doutor em administração, 1 doutor em ciência da informação, 1 mestre em ciências médicas, 1 doutor em biologia, 1 doutor em engenharia elétrica e 1 doutor em psicologia. Faz-se notar que algumas solicitações de inscrição foram indeferidas, pois os candidatos não preenchiam os requisitos solicitados. Além dos matriculados e do professor ministrante, o curso contou com um discente de graduação em informática, bilíngue (português-inglês), que atuou junto ao grupo de participantes na qualidade de monitor.

A visão educacional do curso foi baseada na interatividade e na mobilização de conhecimento dos participantes. Foram empregadas aulas práticas e exercícios práticos individuais, em duplas e/ou em grupos. Quando necessário, os participantes trocaram experiências de pesquisa em língua inglesa, língua francesa e em língua portuguesa. Embora não previsto inicialmente, o multilinguismo foi importante e incrementou a interação do grupo, viabilizando a troca de conhecimentos e experiências em pesquisa.

Todos os materiais didáticos do curso, incluindo textos, exercícios e avaliações foram disponibilizados e acessados pelos participantes via Internet por meio de uma plataforma Wiki. O uso dessa plataforma foi importante, sobretudo, para que cada participante do curso tivesse um 
feedback diário do professor ministrante acerca de cada atividade realizada. Assim, seu uso facilitou a avaliação permanente da formação dos participantes, bem como tornou o curso fisicamente "mais leve", na medida em que seria cansativo imprimir e transportar todos os textos e exercícios empregados no curso no suporte papel.

Todos os participantes tinham habilidades para o uso do computador. Mesmo assim, o discente monitor foi requisitado em alguns momentos por 3 participantes (um do campo da enfermagem, um do campo da administração e um do campo da medicina) para auxílio no download e upload de arquivos. Assim, a presença do discente monitor na sala foi relevante.

Os participantes foram convidados a avaliar o curso ministrado em dois momentos: ao final do segundo dia e ao final do quinto dia de curso. Essas avaliações não foram obrigatórias, mas foi informado aos participantes de que suas manifestações serviriam para aperfeiçoar futuras ofertas do curso.

Como mostrado na tabela 1 e nos quadros 1 e 2, a avaliação das aulas relacionadas aos métodos mistos foi positiva. Numa perspectiva quantitativa, esse conteúdo recebeu dos participantes uma média superior a 4 (numa escala de 1 a 5 , onde 1 seria ruim e 5 excelente) em todos os itens avaliados. Numa perspectiva qualitativa, os comentários efetuados pelos participantes indicam um empoderamento em pesquisa e uma aquisição de conhecimentos relevantes relacionados à avaliação da qualidade de pesquisas e estudos que empreguem métodos mistos.

Tabela 1 - Avaliação dos participantes sobre as aulas relacionadas aos métodos mistos

\begin{tabular}{|lc|}
\hline Aspectos avaliados & Média (n=11) \\
& $\begin{array}{c}\text { De 1(ruim) a } \\
\text { 5 (excelente) }\end{array}$ \\
Os dois primeiros dias do curso foram pertinentes ao seu trabalho? & 4.5 \\
O nível da aula foi apropriado? & 4.4 \\
O nível das atividades práticas foi apropriado? & 4.1 \\
A competência do professor foi adequada? & 5.0 \\
Como foi o uso de recursos audiovisuais pelo professor? & 4.7 \\
O seu entendimento sobre métodos mistos cresceu? & 4.1 \\
Você aprendeu algum conhecimento novo? & 4.5 \\
Os dois primeiros dias do curso incrementaram sua prática de pesquisa? & 4.1 \\
\hline
\end{tabular}


Quadro 1 - Comentários dos participantes sobre os aspectos que mais gostaram nas aulas relacionadas aos métodos mistos

\section{Comentários}

- A qualidade das atividades práticas em sala de aula.

- Eu gostei da maneira como os diferentes métodos quantitativos, qualitativos e mistos foram apresentados.

- As estratégias didáticas, os exemplos práticos e os exercícios, e a possibilidade de discutir algumas questões em língua portuguesa.

- A possibilidade de interagir e aprender com o professor e com meus colegas.

- A ferramenta de análise crítica dos métodos mistos foi um dos tópicos mais importantes. Eu já tinha usado esse tipo de ferramenta, mas não conhecia esse que foi apresentado aqui.

- O curso me ajudou a melhor entender como eu posso fazer uma boa e rigorosa pesquisa usando métodos mistos.

- Eu estou mais preparada para entender e criticar os estudos que empregam métodos mistos.

Como evidenciam os comentários anteriores, há que se observar que os exercícios práticos empregando os conceitos apreendidos são fundamentais para maior assimilação dos conteúdos. Dessa forma, reforça-se a necessidade da educação continuada, que não parece ser suprida apenas com a leitura de manuais metodológicos.

Quadro 2 - Comentários dos participantes sobre os futuros usos dos conhecimentos adquiridos referente aos métodos mistos

\section{Comentários}

- Eu ensino metodologia e eu estou planejando melhorar minhas aulas após esse treinamento.

- Eu reverei meu projeto de pesquisa.

- Eu desenvolverei minha pesquisa de pós-doutorado usando métodos mistos.

- Eu não tenho experiência no uso de métodos mistos, mas esse treinamento me motivou a tentar fazer esse tipo de estudo.

- Eu entenderei melhor as pesquisas que usam métodos mistos e as revisões mistas.

- Eu encontrei muitas possibilidades de trabalhar os dados.

- Eu pensarei em um projeto de pesquisa usando métodos mistos porque eu, de fato, posso melhor entendê-los.

- Quando eu for professor, eu começarei meu grupo de pesquisa usando métodos mistos. - Agora, eu tenho uma nova perspectiva e novas ideias para desenvolver meus futuros estudos empregando métodos mistos.

Os comentários apresentados no quadro 2 ilustram que o interesse pela mudança da ação em pesquisa foi motivado após o processo de educação continuada, reforçando a sua importância como um recurso de caráter emancipatório.

Como mostrado na tabela 2 e no quadro 3, a avaliação das aulas relacionadas com as revisões mistas foi igualmente positiva. Numa perspectiva quantitativa, esse conteúdo recebeu 
dos participantes uma média superior a 3,9 em todos os itens avaliados. Numa perspectiva qualitativa, os comentários efetuados pelos participantes evidenciaram que conhecem pouco essa temática.

Tabela 2 - Avaliação dos participantes sobre as aulas relacionadas às revisões mistas

\begin{tabular}{|lc|}
\hline Aspectos avaliados & $\begin{array}{c}\text { Média (n=10) } \\
\text { De 1 (ruim) a 5 (excelente) }\end{array}$ \\
Os dois últimos dias do curso foram pertinentes ao seu trabalho? & 4.5 \\
O nível da aula foi apropriado? & 4.6 \\
O nível das atividades práticas foi apropriado? & 4.3 \\
A competência do professor foi adequada? & 5 \\
Como foi o uso de recursos audiovisuais pelo professor? & 4.8 \\
O seu entendimento sobre revisões mistas cresceu? & 4.3 \\
Você aprendeu algum conhecimento novo? & 4.5 \\
Os dois últimos dias do curso incrementaram sua prática de pesquisa? & 3.9 \\
\hline
\end{tabular}

Quadro 3 - Comentários dos participantes sobre as aulas relacionadas às revisões mistas

\section{Comentários}

- Foi a primeira vez que ouvi falar em revisões mistas.

- Esta foi a minha primeira experiência com a temática revisões mistas.

- O curso me fez refletir sobre a necessidade de ser conhecedor em revisões mistas.

- O curso foi totalmente novo e inovador.

- Este foi o meu primeiro contato com revisões mistas, e foi maravilhoso!

- Eu conheço muito pouco de revisões mistas, comparado com o conhecimento que tenho sobre pesquisas que empregam métodos mistos.

Em linhas gerais, a realização desse curso evidenciou a importância da educação continuada em metodologias de pesquisa para os pesquisadores brasileiros a fim de que possam exercer maximamente seu potencial criativo e inovador, competindo por publicações de forma menos prejudicada no cenário internacional. 


\section{Conclusão} critérios de avaliação

$\mathrm{Na}$ tentativa de apresentar janelas de oportunidades científicas, este trabalho sistematizou conceitos e critérios de avaliação relacionados à produção de pesquisas empregando métodos mistos e revisões mistas. Ao longo do trabalho, imagina-se que o leitor tenha observado que a produção de pesquisa com novos desenhos exige uma educação continuada do pesquisador. Esse aspecto foi explicitado pelo estudo de caso apresentado que relata uma experiência de ensino-aprendizagem envolvendo métodos mistos e revisões mistas para pesquisadores brasileiros.

Entende-se que a oferta de cursos de educação continuada a pesquisadores sobre métodos de pesquisa mistos e revisões de literatura mistas permite aumentar a capacidade de investigação nacional e internacional, ampliar a massa crítica de revisores experientes em métodos mistos e revisões de literatura mistas para atuação em agências de fomento nacionais e internacionais, bem como para atuação em periódicos nacionais e internacionais, e, reforçar o potencial de colaboração internacional para desenvolvimento de projetos de pesquisa.

Imagina-se que, após o relato aqui apresentado, outras oportunidades de educação continuada em métodos mistos surgirão e, com certeza, beneficiarão estudantes de pósgraduação em meados ou final de doutorado, recém-doutores e pesquisadores independentes que queiram se aperfeiçoar no conhecimento de protocolos de pesquisa e de publicação envolvendo métodos mistos. Especificamente para o campo da ciência da informação, há que se destacar que a produção de revisões mistas de literatura coloca uma demanda por profissionais da informação e pesquisadores desse campo que tenham conhecimentos em métodos de pesquisa mistos e revisões de literatura mistas. 


\section{Referências}

BAHEIRAEI, A. et. al. Iranian adolescents' insufficient physical activity: a mixed methods explanatory sequential study. International Journal of Adolescent Medicine and Health, v. 28, n. 1, p. 79-89, 2016.

BÉLANGER, E. et al. Shared decision-making in palliative care: a systematic mixed studies review using narrative synthesis. Palliative Medicine, v. 25, n. 3, p. 242-261, 2011.

BORNMANN, L. et al. In public peer review of submitted manuscripts, how do reviewer comments differ from comments written by interested members of the scientific community? A content analysis of comments written for Atmospheric Chemistry and Physics.

Scientometrics, v. 93, n. 3, p. 915-929, 2012.

BOYATZIS, R. E. Transforming qualitative information. Thousand Oaks: Sage, 1998.

BRYMAN, A. Integrating quantitative and qualitative research: how is it done? Qualitative Research, v. 6, n. 1, p. 97-113, 2006.

BUELENS, M. et al. Methodological issues in negotiation research: a state of the art review. Group Decision and Negotiation, v. 17, n. 4, p. 321-345, 2008.

CABRERA, N. L. Using a sequential exploratory mixed-method design to examine racial hyperprivilege in higher education. In: GRIFFIN, K. A. et al. New directions for institutional research: using mixed-methods approaches to study intersectionality in higher education. San Francisco: Jossey-Bass, 2011. p. 77-91.

CRESWELL, J. W.; CLARK, V. L. P. Designing and conducting mixed methods research. Thousand Oaks: Sage, 2010.

FIDEL, R. Are we there yet? mixed methods research in library and information science. Library \& Information Science Research, v. 30, n. 4, p. 265-272, 2008.

HARDEN, A. et al. Teenage pregnancy and social disadvantage: systematic review integrating controlled trials and qualitative studies. BMJ, 339. 2009

JOHNSON, R. B.; ONWUEGBUZIE, A. J.; TURNER, L. A. Toward a definition of mixed methods research. Journal of Mixed Methods Research, v. 1, n. 2, 2007, p.112-133.

JONES, S. Alternative perspectives of safety in home delivered health care: a sequential exploratory mixed method study. Journal of Advanced Nursing, v. 72, n. 10, p. 2536-2546, 2016.

KING, N. et al. Determinants of primary school non-enrollment and absenteeism: results from a retrospective, convergent mixed methods, cohort study in rural Western Kenya. PloS One, v. 10, n. 9, p. e0138362, 2015.

LADLE, R. J.; TODD, P. A.; MALHADO, A. C. M. Assessing insularity in global science. Scientometrics, v. 93, n. 3, p. 745-750, 2012.

MA, L. Some philosophical considerations in using mixed methods in library and information 
science research. Journal of the American Society for Information Science and Technology, v. 63, n. 9, p. 1859-1867, 2012.

MERTENS, D. M. et al. Expanding thinking through a kaleidoscopic look into the future: implications of the mixed methods international research association's task force report on the future of mixed methods. Journal of Mixed Methods Research, v. 10, n. 3, p. 221-227, 2016.

MILLS, E. J. et al. Barriers to participation in clinical trials of cancer: a meta-analysis and systematic review of patient-reported factors. The Lancet Oncology, v. 7, n. 2, p. 141-148, 2006.

MISHNA, F. et al. Prevalence, motivations, and social, mental health and health consequences of cyberbullying among school-aged children and youth: protocol of a longitudinal and multi-perspective mixed method study. JMIR Research Protocols, v.5, n, 2, e83, 2016.

NEUENDORF, K. A. The content analysis: guidebook. Thousand Oaks: Sage, 2002.

O'CATHAIN, A.; MURPHY, E.; NICHOLL, J. The quality of mixed methods studies in health services research. Journal of Health Services Research and Policy, v. 13, n. 2, p. 9298, 2008.

O'CONNOR, S. J. Peer review: problem or solution in relation to publication bias, transparency and the internationalisation of scientific research outputs? European Journal of Cancer Care, v. 21, n. 6, p. 701-702, 2012.

PACE, R. et al. Testing the reliability and efficiency of the pilot Mixed Methods Appraisal Tool (MMAT) for systematic mixed studies review. International Journal of Nursing Studies, v. 49, p. 47-53, 2012.

PLUYE, P. Les méthodes mixtes. In: RIDDE, V.; DAGENAIS, C. (Ed.). Approches et pratiques en évaluation de programme. Montréal: Presses de l'Université de Montréal, 2012. p. 125-143.

PLUYE, P. et al. The impact of clinical information-retrieval technology on physicians: a literature review of quantitative, qualitative and mixed-method studies. International Journal of Medical Informatics, v. 74, n. 9, p. 745-768, 2005.

A scoring system for appraising mixed methods research, and concomitantly appraising qualitative, quantitative and mixed methods studies in mixed studies reviews. International Journal of Nursing Studies, v. 46, p. 522-546, 2009a.

Understanding divergence of quantitative and qualitative data (or results) in mixed methods studies. International Journal of Multiple Research Approaches, v. 3, n. 1, p.5872, 2009b.

Proposal: a mixed methods appraisal tool for systematic mixed studies reviews.

Montreal: McGill University, 2011. Disponível em:

<http://mixedmethodsappraisaltoolpublic.pbworks.com>. Acesso em:

POPE, C.; MAYS, N.; POPAY, J. Synthesizing quantitative and qualitative health

InCID: R. Ci. Inf. e Doc., Ribeirão Preto, v. 8, n. 2, p. 4-24, set. 2017/fev. 2018. 
research. Adelaide: Ramsay Books, 2007.

PORTA, M. A dictionary of epidemiology. Oxford: Oxford University Press, 2008.

SCHWANDT, T. A. Dictionary of qualitative inquiry. Thousand Oaks: Sage, 2007.

SPIGT, M.; ARTS, I. C. W. How to review a manuscript. Journal of Clinical Epidemiology, v. 63, p. 1385-1390, 2010.

THOMAS, J. et al. Integrating qualitative research with trials in systematic reviews. BMJ, v. 328, n. 7446, p. 1010-1012, 2004.

WAKIMOTO, D. K. Information literacy instruction assessment and improvement through evidence based practice: A mixed method study. Evidence Based Library and Information Practice, v. 5, n. 1, p. 82-92, 2010.

WINCK, J. C. et al. To publish or perish: how to review a manuscript. Revista Portuguesa de Pneumologia, v. 17, n. 2, p. 96-103, 2011.

YI, Y. J. Health literacy and health information behavior of Florida public library users: A mixed methods study. Journal of Librarianship \& Information Science, v. 47, n. 1, p. 17 29, 2015. 